\title{
PATHOGENESIS OF CONGENITAL ADHESIONS: CYTOKINES AND HUMAN $\beta$-DEFENSIN 2
}

\author{
Aleksejs Zavorins ${ }^{1}$, Mara Pilmane $^{1}$, Olafs Volrats ${ }^{2}$ \\ ${ }^{1}$ Institute of Anatomy and Anthropology, Riga Stradiņš University, Latvia \\ ${ }^{2}$ University Children's Hospital, Pediatric surgery, Riga, Latvia
}

\begin{abstract}
Intestinal malrotation is a failure of the bowel to attain the correct position during $5^{\text {th }}$ to $10^{\text {th }}$ week of embryogenesis that results in fixation of caecum to the upper part on the right side of abdominal wall with congenital peritoneal adhesions also known as Ladd's bands. There are several factors speculated to have an impact on formation of the congenital adhesions, including intrauterine mesothelial trauma due to ischemia or an infectious agent. Therefore, the aim was to research expression of local pro- and anti-inflammatory factors, ischemia related factors and antimicrobial peptides in the tissue of congenital adhesions.

Biopsy samples of congenital peritoneal adhesions from 20 neonates that were 0 to 4 days old were gathered from the collection of Institute of Anatomy and Anthropology of Riga Stradins University. All biopsy samples were obtained during a primary laparotomy due to obstructive gut malrotation. Tissue samples were stained with hematoxylin and eosin, as well as immunohistochemical staining with antibodies to VEGF, IL-1, IL-6, IL-10 and human $\beta$-defensin 2 was also performed.

Relatively increased quantity of IL-10, IL- 6 and human $\beta$-defensin 2 was discovered, that suggests an activation of an anti-inflammatory response, possibly due to an intrauterine infection. Additionally, a relatively increased amount of IL-10 and IL-6 positive macrophages and fibroblasts suggests that a persistant inflammation triggered development of fibrous tissue. Increased quantity of human $\beta$-defensin 2 positive and decreased quantity of VEGF positive endothelial cell suggests that angiogenesis was mediated by $\beta$-defensin 2 independentaly from VEGF pathway.
\end{abstract}

Keywords: congenital peritoneal adhesions, Ladd's bands, human $\beta$-defensin 2, IL-10, VEGF, IL-6 


\section{INTRODUCTION}

Intestinal malrotation is a failure of the bowel to attain the correct position during $5^{\text {th }}$ to $10^{\text {th }}$ week of embryogenesis that results in fixation of caecum to the upper part on the right side of abdominal wall with peritoneal adhesions also known as Ladd's bands $[20,24]$. The reported incidence of intestinal malrotation is approximately 1 case per 500 births. In up to $80 \%$ it presents itself during the first month of life as bile vomiting of the newborn due to a duodenal obstruction [8]. Therefore, this condition requires an urgent surgical intervention [16]. Unfortunately, there are sparse publications that show the possible aetiology and pathogenesis of the congenital peritoneal adhesions. There are several factors speculated to have an impact on formation of the congenital adhesions, including genetic defects that impair intestinal embryogenesis and intrauterine mesothelial trauma due to ischemia or an infectious agent $[1,5,20]$.

Formation of peritoneal adhesions is a result of a synergic impact of three factors on the peritoneum - mesothelial trauma, hypoxia and inflammation [2]. Mesothelial injury is followed by a local inflammation, increased vascular permeability and exudation of fibrin. The fibrinous clot is an essential scaffold for peritoneal repair that promotes mesothelial migration. However, persistence of the fibrin due to impaired fibrinolysis leads to migration of fibroblasts followed by synthesis of extracellular matrix (ECM), vascular ingrowth and formation of peritoneal adhesions [22]. Fibrinolysis can normally be achieved by plasmin that is produced from plasminogen. Production of plasmin is upregulated by tissue plasminogen activator (tPA) and downregulated by plasminogen activator inhibitor - 1 (PAI-1) [2, 22]. Impaired balance between these systems that can be provoked by hypoxia and inflammation and it is a pivotal factor in adhesiogenesis.

The inflammatory response involves fibroblasts and macrophages recruited into the fibrinous clot, as well as mesothelial cells that secrete several cytokines, including tumor growth factor- $\beta 1$ (TGF- $\beta 1$ ), tumor necrosis factor- $\alpha$ (TNF- $\alpha$ ), IL-1 and IL- $6[15,22,27]$. TGF- $\beta 1$, TNF- $\alpha$, IL- 1 and IL- 6 are pro-inflammatory cytokines that are associated with increased secretion of PAI- 1 and reduced release of tPA. TGF- $\beta 1$ can additionally stimulate collagen and fibronectin synthesis by fibroblasts and inhibit ECM resorption $[2,22]$.

IL-10 is an anti-inflammatory cytokine that is expressed by macrophages and lymphocytes [23]. Production of IL-10 is dependent on many factors and can be mediated through Toll-like receptors (TLR). TLRs are a part of an innate immune system that recognize several types of signals and modulate 
the function of inflammatory cells [12]. These signals include pathogen-associated molecular patterns (PAMPs) and danger-associated molecular patterns (DAMPs) $[4,10]$. DAMPs are endogenous mediators that are released from necrotic cells. In an in vivo experiment TLR 4 in response to DAMPs in a sterile wound induced production of IL-10. This was associated with inhibition of TGF $\beta$ and thus reduced collagen production and reduced neoangiogenesis [4, 14]. However, PAMPs consist mainly of structural components of infectious agents, including lipopolysaccharides (LPS) of Gram negative bacteria and major outer membrane protein (MOMP) of Chlamydia spp.. TLRs in response to LPSs are capable of inducing simultaneous production of IL-10 and several pro-inflammatory cytokines. Subsequently, IL-10 suppresses responses of T helper 1 lymphocytes $\left(\mathrm{Th}_{1}\right)$ by inhibiting production of TNF $\alpha$ and INF $\gamma$. However, it enables $\mathrm{Th}_{2}$ responses that include production of IL-6 [7]. In an in vivo model of a bacterial peritonitis a persistent inflammation induced IL-6 mediated peritoneal fibrosis [9].

Hypoxia is associated with increased production of vascular endothelial growth factor (VEGF) in the endothelium through activation of hypoxia inducible factor - $1 \alpha$ (HIF-1 $\alpha$ ) in the fibroblasts and mesothelial cells. VEGF induces angiogenesis by remodeling vascular basement membrane and promoting migration and proliferation of endothelial cells [22, 25]. Hypoxia inhibits apoptosis and has several additional effects on the fibroblasts that promote adhesiogenesis $[2,22]$. However, there is an alternative Human $\beta$-defensin 2 (hBD 2) dependent pathway that also promotes angiogenesis $[3,13]$.

Human $\beta$-defensin 2 (hBD 2) is an antimicrobial peptide that is a part of the innate immune system and is predominantly expressed by keratinocytes, epithelium and mesothelium. Expression of hBD 2 is induced by LPS through TLR, additionally inflammatory stimuli such as IL-1 $\beta$ and TNFa also upregulate hBD 2 expression [10]. Besides a well-known bactericidal activity, hBD 2 is a chemoattractant of macrophages and neutrophils, and is also considered a pro-angiogenic factor that stimulates migration, proliferation and capillarylike tube formation of endothelial cells, independently from VEGF pathways $[3,13]$. Higher serum levels of hBD 2 also correlate with increased production of INF $\gamma$ and IL-10 by T-cells [11], inversely IL-10 inhibits expression of hBD2 [6], thus a negative feedback mechanism is plausible.

On the basis of all of the above mentioned the aim of our work was to research expression of local pro- and anti-inflammatory factors, ischemia related factors and antimicrobial peptides in congenital adhesion. 


\section{MATERIALS AND METHODS}

Biopsy samples of congenital peritoneal adhesions from 20 neonates that were 0 to 4 days old were gathered from the collection of Institute of Anatomy and Anthropology of Riga Stradins University. All biopsy samples were obtained during a primary laparotomy due to obstructive gut malrotation and several additional pathologies (see Table 1). Permission was granted by the Ethics Committee of Riga Stradins University on March 12 2007.

Biopsy samples were fixed in Stefanini's solution and embedded in paraffin, $4 \mu \mathrm{m}$ thick sections were stained with hematoxylin and eosin, subsequently immunohistochemical staining was performed with antibodies to vascular endothelial growth factor (anti-VEGF, 1:50 solution, Santa Cruz, USA), interleukin-1 (anti-IL-1, 1:50 solution, Santa Cruz, USA), interleukin-6 (anti-IL-6, 1:50 solution, Santa Cruz, USA), interleukin-10 (anti-IL-10, 1:400 solution, Abcam, England), PGP 9.5 (anti-PGP 9.5, 1:200 solution, Invitrogen, USA) and $\beta$-defensin 2 (anti $\beta$-defensine 2, 1:100 solution, R\&D systems, Germany).

Stained samples were microscoped and photographed at 200X, 250X, 400X magnification, and evaluated using semi-quantitative method. Depending on the relative quantity of positively stained structures, specimens were rated as having a few positive structures $(+)$, moderate quantity of positive structures $(++)$, numerous quantity of positive structures $(+++)$ and an abundant amount of positive structures $(++++)$ [Pilmane M, 1998].

Data analysis was conducted using Statistical Package for the Social Sciences (SPSS) program version 20.0. Results of semi-quantitative evaluation were transformed into numerical form, where + is equal to $1,++$ is equal to 2 , +++ is equal to $3,++++$ is equal to 4 and a negative result is equal to 0 . Nonparametric statistics were used and Spearman's rank correlation coefficient was calculated to compare co-expression of the markers in the biopsies. Twotailed $\mathrm{P}$ values of $\leq 0.05$ were considered as statistically significant.

\section{RESULTS}

Congenital adhesions revealed neoangiogenesis and slightly round fibroblasts, a perivascular inflammatory infiltrate (Fig. 1), as well as fibrinous deposits and formation of polynuclear epithelioid cells (Fig. 2).

Immunohistochemical staining (Table 1) of the congenital adhesions samples revealed a few (+) VEGF positive endotheliocytes (Fig. 3), as well as a few (+) IL-1 positive macrophages (Figure 4). In 3 samples IL-1 positive structures were absent. Few to moderate quantity (+/++) of IL- 6 and IL-10 positive macrophages and fibroblasts were present in congenital adhesion samples (Figure 5 
and 6). Moderate to numerous number $(++/+++)$ of human $\beta$-defensin 2 positive endotheliocytes, myocytes and macrophages were also observed (Figure 7).

There was no correlation identified (Table 2) with Spearman's Rank test between IL-10 and human beta defensin $2\left(\mathrm{r}_{\mathrm{s}}[20]=-0,066, \mathrm{p}=0,784\right)$.

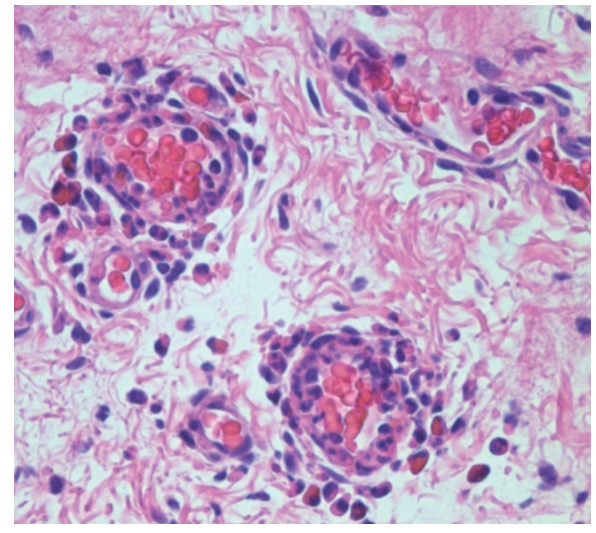

Figure 1. Perivascular inflammation in a congenital adhesions patient 5, H\&E, 400x.

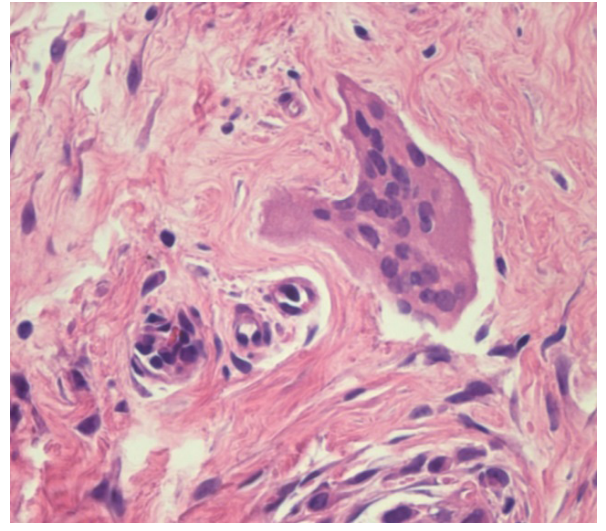

Figure 2. Polynuclear epitheloid cell in a congenital adhesion specimen, patient 11 , $\mathrm{H} \& \mathrm{E}, 400 \mathrm{x}$

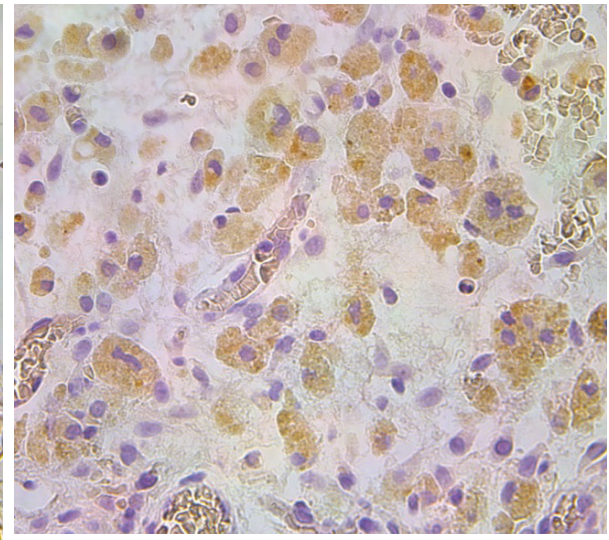

Figure 4. IL-1 positive epithelioid cells in patient 11, IL-1 IMH, 400x
Figure 3. VEGF positive endothelium in congenital adhesions of patient 14, VEGF $\mathrm{IMH}, 400 \mathrm{x}$ 


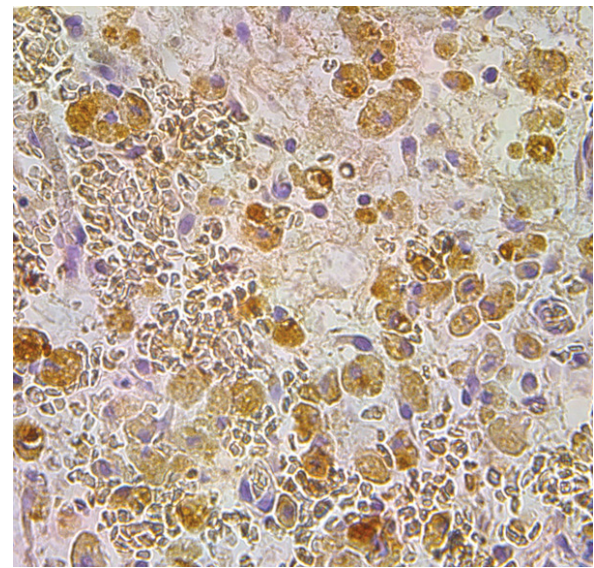

Figure 5. IL-6 positive epithelioid cells in patient 11, IL-6 IMH, 400x

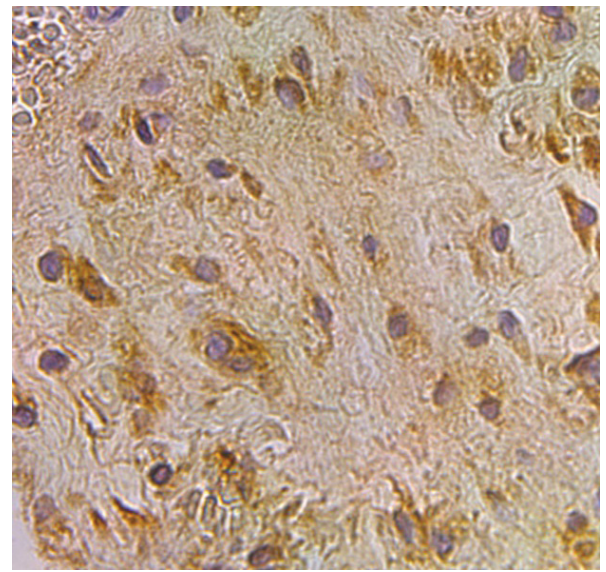

Figure 6. IL-10 positive fibroblasts and macrophages in congenital adhesions of patient 9, IL-10 IMH, 400x

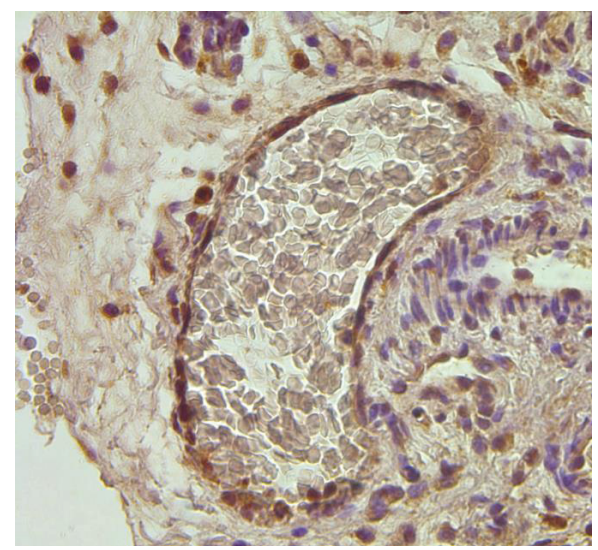

Figure 7. Human $\beta$-defensin 2 positive epithelium in a congenital adhesions specimen, $\mathrm{HBD} 2 \mathrm{IMH}, 400 \mathrm{x}$ 


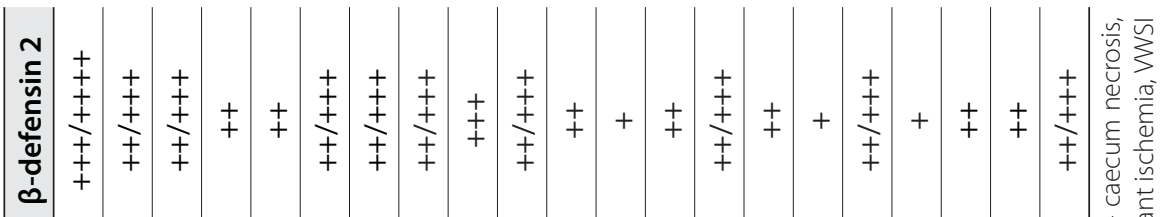

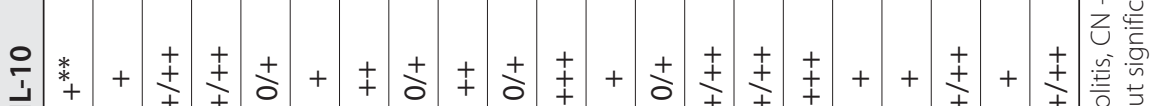

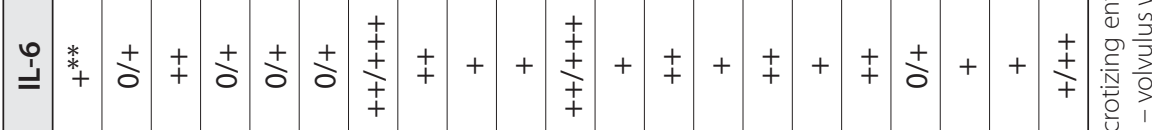

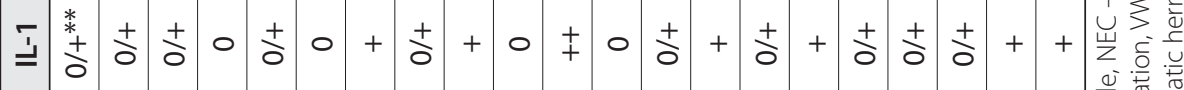

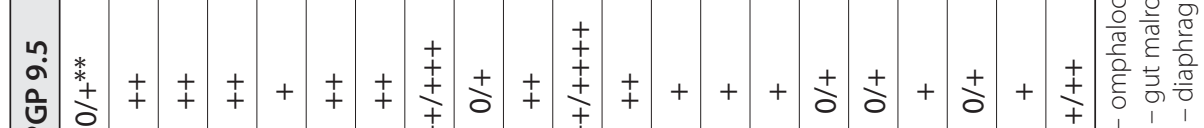

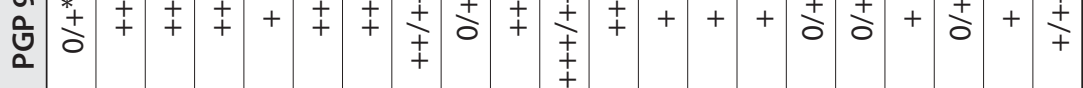


Table 2. Spearman's Rank test correlation between IL-10 and HBD2 in congential adhesion tissue samples.

\begin{tabular}{lcc}
\hline Correlation & $\mathbf{r}_{\mathrm{s}}$ & P value \\
\hline IL-1 and HBD 2 & $-0,066$ & 0,784 \\
\hline
\end{tabular}

\section{DISCUSSION}

The relatively increased quantity of IL-6, IL-10 and HBD 2 positive structures, in comparison to the relatively decreased quantity of IL-1 positive structures, points out to a possible impact of PAMPs mediated through Toll-like receptors $[7,10]$. In other words macrophages and lymphocytes produce IL-6, IL-10 and HBD 2 in response to a possible stimulation by LPS, thus the presented results allow us to speculate that formation of congenital adhesions could be a consequence of an intrauterine exposure to an infectious agent. Previous research has demonstrated that IL-10 is anti-inflammatory cytokine associated with decreased development of fibrosis [2, 22]. Nevertheless, a recent publication demonstrated that IL-10 predominantly inhibits production of TNF $\alpha$ and that expression of IL-6 is less affected [7]. Persistent inflammation and repetitive production of IL-6 is associated with development of peritoneal fibrosis [9]. This is a proposed immunological mechanism for the development of the congenital adhesions. This is also supported by a recent experiment. The in vivo experiment showed that chorioamnionitis associated with intrauterine exposure to LPS leads to impaired gut development due to inflammatory responses mediated through TLRs [28].

The relatively small quantity of VEGF positive structures demonstrates that there was no pronounced hypoxia that could have stimulated neoangiogenesis during the formation of congenital adhesions [25]. However, increased quantity of HBD 2 positive endotheliocytes point to angiogenesis that could have resulted from an impact of LPS on TLR $4[3,13]$.

There was no linear correlation found between IL-10 and HBD 2. The linear correlation between IL-10 and HBD 2 was evaluated due to data that the expression of these factors is dependent upon each other [6, 11]. Increased level of IL-10 to HBD2 ratio could have indicated a negative feedback mechanism exerted by IL-10 on HBD 2 [6]. However, there are several possible explanations our results. Production of IL-10 can be induced by several pathways independently from TLR 4 and HBD 2. Therefore, other unknown factors can modify expression of IL-10 and the relationship between IL-10 and HBD 2. Our patients showed clinical variability. Other researches have also encoun- 
tered a similar trend. Intestinal malrotation and congenital adhesions are often a part of multivariate syndromes, although non-syndromic cases have also been described [20]. Different genetic mutations, including mutation of Foxf1 gene, have been implicated [19]. Therefore, intestinal malrotation and congenital adhesions combine a broad spectrum of diseases with different, possibly multifactorial aetiology, and a comprehensive research of the possible role of intrauterine infection in pathogenesis of the congenital adhesions is appropriate.

\section{CONCLUSIONS}

1. Relatively increased quantity of IL-10, IL-6 and HBD2 suggests an activation of an anti-inflammatory response, possibly due to an intrauterine infection. Additionally, a relatively increased amount of IL-10 and IL-6 positive macrophages and fibroblasts suggests that a persistant inflammation triggered development of fibrous tissue.

2. Increased quantity of human beta defensin 2 positive and decreased quantity of VEGF positive endothelial cell suggests that angiogenesis was mediated by human beta defensin 2 independentaly from VEGF pathway.

\section{REFERENCES}

1. Arung W., Meurisse M., Detry O. Pathophysiology and prevention of postoperative peritoneal adhesions. World J Gastroenterol. 2011 Nov 7; 17(41): 4545-53

2. Atta H.M. Prevention of peritoneal adhesions: a promising role for gene therapy. World J Gastroenterol. 2011 Dec 14;17(46):5049-58

3. Baroni A., Donnarumma G., Paoletti I., Longanesi-Cattani I., Bifulco K., Tufano M.A., Carriero MV. Antimicrobial human beta-defensin-2 stimulates migration, proliferation and tube formation of human umbilical vein endothelial cells. Peptides. 2009 Feb;30(2):267-72.

4. Brancato S.K., Thomay A.A., Daley J.M., Crane M.J., Reichner J.S., Sabo E., Albina J.E. Toll-like receptor 4 signaling regulates the acute local inflammatory response to injury and the fibrosis/neovascularization of sterile wounds. Wound Repair Regen. 2013 Jul-Aug;21(4):624-33. doi: 10.1111/wrr.12061. Epub 2013 Jun 11.

5. Brüggmann D., Tchartchian G., Wallwiener M., Münstedt K., Tinneberg H.R., Hackethal A. Intra-abdominal adhesions: definition, origin, significance in surgical

6. Chen Q.X, Zhou J.L., Book M. IL-10 inhibits inducible expression pattern of beta-defensin-2 in human peripheral blood cells. Zhejiang Da Xue Xue Bao Yi Xue Ban. 2006 Nov;35(6):596-9, 604. 
7. Duell B.L., Tan C.K., Carey A.J., Wu F., Cripps A.W., Ulett G.C. Recent insights into microbial triggers of interleukin-10 production in the host and the impact on infectious disease pathogenesis. FEMS Immunol Med Microbiol. 2012 Apr;64(3):295-313.

8. Durkin E.T., Lund D.P., Shaaban A.F., Schurr M.J., Weber S.M. Age-related differences in diagnosis and morbidity of intestinal malrotation. J Am Coll Surg. 2008 Apr;206(4):658-6

9. Fielding C.A., Jones G.W., McLoughlin R.M., McLeod L., Hammond V.J. Interleukin-6 signaling drives fibrosis in unresolved inflammation. Immunity. 2014 Jan 16;40(1):40-50.

10. Jarczak J., Kościuczuk E.M., Lisowski P., Strzałkowska N., Jóźwik A., Horbańczuk J., Krzyżewski J., Zwierzchowski L., Bagnicka E. Defensins: natural component of human innate immunity. Hum Immunol. 2013. Sep; 74(9):1069-79.

11. Kanda N., Kamata M., Tada Y., Ishikawa T., Sato S., Watanabe S. Human $\beta$-defensin-2 enhances IFN- $\gamma$ and IL-10 production and suppresses IL-17 production in T cells. J Leukoc Biol. 2011 Jun;89(6):935-44. doi: 10.1189/ jlb.0111004. Epub 2011 Mar 2.

12. Kawaratani H., Tsujimoto T., Douhara A., Takaya H., Moriya K., Namisaki T., Noguchi R., Yoshiji H., Fujimoto M., Fukui H. The effect of inflammatory cytokines in alcoholic liver disease. Mediators Inflamm. 2013;2013:495156.

13. Kawsar H.I., Ghosh S.K., Hirsch S.A., Koon H.B., Weinberg A., Jin G. Expression of human beta-defensin-2 in intratumoral vascular endothelium and in endothelial cells induced by transforming growth factor beta. Peptides. 2010 Feb;31(2):195-201.

14. Kieran I., Knock A., Bush J., So K., Metcalfe A., Hobson R., Mason T., O’Kane S., Ferguson M. Interleukin-10 reduces scar formation in both animal and human cutaneous wounds: results of two preclinical and phase II randomized control studies. Wound Repair Regen. 2013 May-Jun;21(3):428-36. doi: 10.1111/ wrr.12043. Epub 2013 Apr 29.

15. Kono H., Onda A., Yanagida T. Molecular determinants of sterile inflammation. Curr Opin Immunol. 2014 Feb;26C:147-156.

16. Lim P.W., Martin N.D., Hicks B.A., Yeo C.J., Cowan S.W. William Edwards Ladd, M.D. (1880-1967): the description of his bands. Am Surg. 2013 Jan;79(1):11-3.

17. Lybeck K.R., Løvoll M., Johansen T.B., Olsen I., Storset A.K., Valheim M. Intestinal strictures, fibrous adhesions and high local interleukin-10 levels in goats infected naturally with Mycobacterium avium subsp. paratuberculosis. J Comp Pathol. 2013 Feb;148(2-3):157-72. doi: 10.1016/j.jcpa.2012.05.007. Epub 2012 Jul 10.

18. Brüggmann D., Tchartchian G., Wallwiener M., Münstedt K., Tinneberg H.R., Hackethal A. Intra-abdominal adhesions: definition, origin, 
significance in surgical practice, and treatment options. Dtsch Arztebl Int. 2010 Nov;107(44):769-75. doi: 10.3238/arztebl.2010.0769. Epub 2010 Nov 5.

19. Mahlapuu M., Ormestad M., Enerbäck S., Carlsson P. The forkhead transcription factor Foxf1 is required for differentiation of extra-embryonic and lateral plate mesoderm. Development. 2001 Jan;128(2):155-66.

20. Martin V., Shaw-Smith C. Review of genetic factors in intestinal malrotation. Pediatr Surg Int. 2010 Aug;26(8):769-81

21. Ramamoorthy S.L., Lee J.K., Luo L., Mintz Y., Cullen J., Easter D.W., Savu M.K., Chock A., Carethers J., Horgan S., Talamini M.A. The inflammatory response in transgastric surgery: gastric content leak leads to localized inflammatory response and higher adhesive disease. Surg Endosc. 2010 Mar;24(3):531-5. doi: 10.1007/s00464-009-0636-7. Epub 2009 Aug 18.

22. Rizzo A., Spedicato M., Mutinati M., Minoia G., Angioni S., Jirillo F., Pantaleo M., Sciorsci R.L. Peritoneal adhesions in human and veterinary medicine: from pathogenesis to therapy. A review. Immunopharmacol Immunotoxicol. 2010 Sep;32(3):481-94. doi: 10.3109/08923970903524367.

23. Saraiva M., O'Garra A. The regulation of IL-10 production by immune cells. Nat Rev Immunol. 2010 Mar;10(3):170-81.

24. Shalaby M.S., Kuti K., Walker G. Intestinal malrotation and volvulus in infants and children. BMJ. 2013 Nov 26;347:f6949.

25. Shibuya M. Vascular endothelial growth factor and its receptor system: physiological functions in angiogenesis and pathological roles in various diseases. J Biochem. 2013 Jan;153(1):13-9.

26. Stankiewicz P., Sen P., Bhatt S.S., Storer M., Xia Z. et al. Genomic and genic deletions of the FOX gene cluster on 16q24.1 and inactivating mutations of FOXF1 cause alveolar capillary dysplasia and other malformations. Am J Hum Genet. 2009 Jun;84(6):780-91. doi: 10.1016/j.ajhg.2009.05.005. Epub 2009 Jun 4.

27. Tanaka T., Kishimoto T. Targeting interleukin-6: all the way to treat autoimmune and inflammatory diseases. Int J Biol Sci. 2012;8(9):1227-36.

28. Wolfs T.G, Kramer B.W., Thuijls G., Kemp M.W., Saito M., Willems M.G., Senthamarai-Kannan P., Newnham J.P., Jobe A.H., Kallapur S.G. Chorioamnionitis-induced fetal gut injury is mediated by direct gut exposure of inflammatory mediators or by lung inflammation. Am J Physiol Gastrointest Liver Physiol. 2014 Mar 1;306(5):G382-93. doi: 10.1152/ajpgi.00260.2013. Epub 2014 Jan 23.

29. Yuan C., Chen W.X., Zhu J.S., Chen N.W., Lu Y.M., Ou Y.X., Chen HQ. IL-10 treatment is associated with prohibitin expression in the Crohn's disease intestinal fibrosis mouse model. Mediators Inflamm. 2013;2013:617145. doi: 10.1155/2013/617145. Epub 2013 Apr 14. 
Address of correspondence:

Aleksejs Zavorins,

Anatomijas un antropologijas institūts,

Kronvalda bulv. 9, Rīga, Latvija, LV-1010

E-mail: aleksejs.zavorins@gmail.com 